\title{
Marine Leisure Sports Based on Realistic VR System for BLeisure Busan
}

\author{
Kim Cheeyong ${ }^{1}$, Jong-Chan Kim², Se-Hoon Jung ${ }^{3}$ and Eung-Kon Kim ${ }^{2 *}$ \\ ${ }^{1}$ College of ICT, Major of Game Animation Engineering, Doug-Eui University \\ ${ }^{2}$ Dept. of Computer Engineering, Sunchon National University \\ ${ }^{3}$ Dept. of Multimedia Engineering, Sunchon National University \\ ${ }^{1}$ kimchee@deu.ac.kr, ${ }^{2}$ seaghost@sunchon.ac.kr, ${ }^{3}$ iam1710@hanmail.net \\ ${ }^{2}$ kek@sunchon.ac.kr
}

\begin{abstract}
Virtual reality (VR) content is focused on next-generation display technology, and offers a strong market for software projects directly related to quality of life, such as medical care. Information and communications technologies (ICT) and convergence technology have evolved at a fast pace, with VR being applied to major areas of business and being adopted in a variety of industries, such as games, movies, and education. In the meanwhile, people's interests in marine leisure sports in the Busan region are extending, domestically as well as from overseas. Therefore, it is necessary to conduct a study on novel leisure sports VR systems, building on the policy of promoting tourism in Busan.

In this paper, existing works related to VR are researched first. For VR-based Busan marine leisure sports to which tangible cultural tour content in the Busan region is applied, the marketing potential will be estimated. This study aims at convergence of the tourism industry, ICT, and a tangible VR platform, which is expected to contribute to fostering glocal future creative industries, bringing region-specific technology settlement, local employment creation, and the fostering of culture.
\end{abstract}

Keywords: Virtual Reality (VR); Ocean Leisure Sports; Business-Leisure (BLeisure), Actural Feeling; Tourism

\section{Introduction}

As convergence-related technology that combines information technology (IT) with our daily lives has advanced rapidly, attention paid to augmented reality (AR) and virtual reality (VR) increases, and the corresponding market scale also shows exponential growth. Globally, the VR market is expected to increase tenfold, from US\$6.7 billion in 2017 to US\$70 billion in 2020. Domestically, academic research into AR is actively conducted, and application fields in which research achievements are adopted are being extended. This explains the change in adopted emerging technologies released by Gartner in $2014[1,2]$.

Virtual world technology is rapidly being replaced with VR technology, and VR is reaching a slope of enlightenment. Due to the changes occurring in tour patterns used to focus on experiences, demand for the study of culture tourism industry content is emerging. Under these circumstances, it is necessary to provide a tangible VR tour service with a new concept, giving the synesthesia experience felt by tourists highest priority [3,5].

Korea signed a memorandum of understanding to promote the VR business with China, which is the country with the biggest demand for VR technology. The Ministry of Science, ICT and Future Planning (MSIP) of Korea has embarked on flagship VR projects

Received (May 23, 2017), Review Result (August 25, 2017), Accepted (September 28, 2017) 
and enforced CNPD policy. The city of Busan, where the proposed system in this paper will be applied, is being driven towards the business-leisure (BLeisure) tourism industry due to its regional specialty. In Busan, a mid- and long-term roadmap for the culture tourism industry is being established, while $\mathrm{R} \& \mathrm{D}$ for the content industry is underway after categorizing it into various fields, including games, video/new media, VR, performance/exhibitions, convergence, and public cultural services.

In this paper, existing works related to VR will be researched first. For VR-based Busan marine leisure sports to which tangible cultural tour contents of the Busan region are applied, the marketing potential will be estimated. Agreeing with the tourism-promotion policy announced by the Busan local government, this paper proposes implementing a tangible VR glocal tourism marine leisure sports platform by converging the tourism industry with the VR industry.

\section{Related Works}

\subsection{Virtual Reality}

VR is defined as a virtually produced environment where users are allowed to experience what they have not experienced before or conditions in non-real and virtual space. VR has several characteristics. First is 3D spaciousness, which is a characteristic that makes the perceiver feel similar interactions and experiences in physical space. Second is that VR is actually flat 2D video, but users feel like they are in the screen owing to the optical illusion created by showing the proper images to the left and right at the same time. Third is a characteristic that induces indirect experiences and feelings about the content by progressing the activities that can be performed in virtual space by means of various controllers $[3,6,7]$.

Table 1. Fields of VR Applications and use Cases

\begin{tabular}{|c|l|}
\hline Field & \multicolumn{1}{c|}{ Use cases } \\
\hline Game & $\begin{array}{l}\cdot \text { Useful for improving a sense of immersion in a first-person point of view } \\
\text { game along with a VR headset and motion recognition technology } \\
\cdot \text { Amplifies spatial effect felt by users while they play various genres of } \\
\text { games, such as FPS, adventure, and horror }\end{array}$ \\
\hline Movie & $\begin{array}{l}\cdot \text { Provides realistic experiences by implementing a scene in the movie using } \\
\text { VR technology } \\
\text { Enables implementing interactive cinema that may change as users make } \\
\text { choices while they participate in the cinema wearing a VR device }\end{array}$ \\
\hline Education & $\begin{array}{l}\cdot \text { Runs classes in a virtual classroom anytime from anywhere by a variety of } \\
\text { students } \\
\cdot \text { Provides science learning from experience by implementing complicated } \\
\text { laws of physics through VR }\end{array}$ \\
\hline Exhibition & $\begin{array}{l}\cdot \text { Creates virtual spaces mimicking distinguished museum and exhibition } \\
\text { centers in the world } \\
\cdot \text { Offers virtual exhibitions to show works or paintings for which exhibitions } \\
\text { have been discontinued in the real world }\end{array}$ \\
\hline SNS & $\begin{array}{l}\cdot \text { Converges tangible technology using a headset and motion recognition } \\
\text { sensors in the SNS space, which is supposed to be implemented based on } \\
\text { virtual space similar to Second Life }\end{array}$ \\
\hline
\end{tabular}




\begin{tabular}{|c|l|}
\hline Healthcare & $\begin{array}{l}\cdot \text { Utilizes VR technology for remote diagnosis and counselling so that users } \\
\text { can get medical treatment at home } \\
\cdot \text { Provides experiences to treat trauma using VR technology in the fields of } \\
\text { psychotherapy and dementia therapy }\end{array}$ \\
\hline Office work & $\begin{array}{l}\text { Provides simulations necessary for constructing a building } \\
\text { Enables remote meetings with distant peers in a virtual space }\end{array}$ \\
\hline
\end{tabular}

VR is an attractive market that worldwide IT companies have strong interest in. In a situation where PCs and mobile handsets face limited growth, VR is considered a future cash cow for the technology industry. The National Information Society Agency (NIA) expects the worldwide VR market to grow to US\$391 billion (426 trillion Korean won [KRW]), and representative global IT companies such as Apple, Google, Samsung, Sony, and HTC are going to move fast towards VR. Table 1 shows application fields and cases in which VR technology can be applied. VR is being adopted to generate virtual reality in a wide variety of environments, including games, movies, education, exhibitions, social networking sites (SNSs), healthcare and office work. In particular, VR technology contributes to making realistic videos in the movie industry in several ways for virtual movie theaters by using headsets and producing movies using VR technology.

Though lots of attention is being paid to VR technology as a next-generation display technology in experience-type content areas, it also has high marketability even in the quality-of-life-related software industry, like medicine, education, and tourism. For example, Figure 1 is from "Titans of Space" providing indirect experiences in a tour of planets in space. It can be utilized for training purposes in various ways, such as military training and steering training. It can also be applied to experience-type contents for architecture design and military/aircraft experiments.
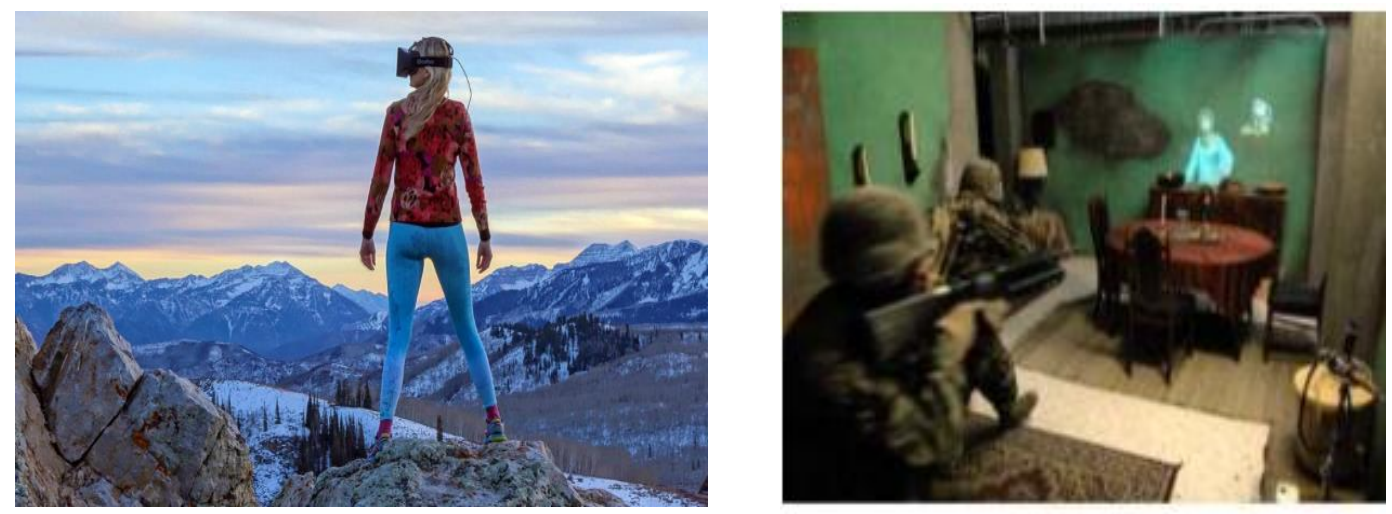

Figure 1. Tangible Contents 


\subsection{Augmented Reality}

Augmented reality is a technique that inserts a virtual object into the visible real world. It is also called mixed reality (MR) because it presents the merging of virtual and real worlds through live streaming; it presents one live projection mixing additional virtual images with the real world. The first research into this see-through technology came by means of the head mounted display (HMD) invented by Ivan Sutherland. Later, Ronald Azuma's design combined images of real-world elements and virtual reality so that it was possible to interact in real time.

Table 2. Main Technologies within the Augmented Reality Field

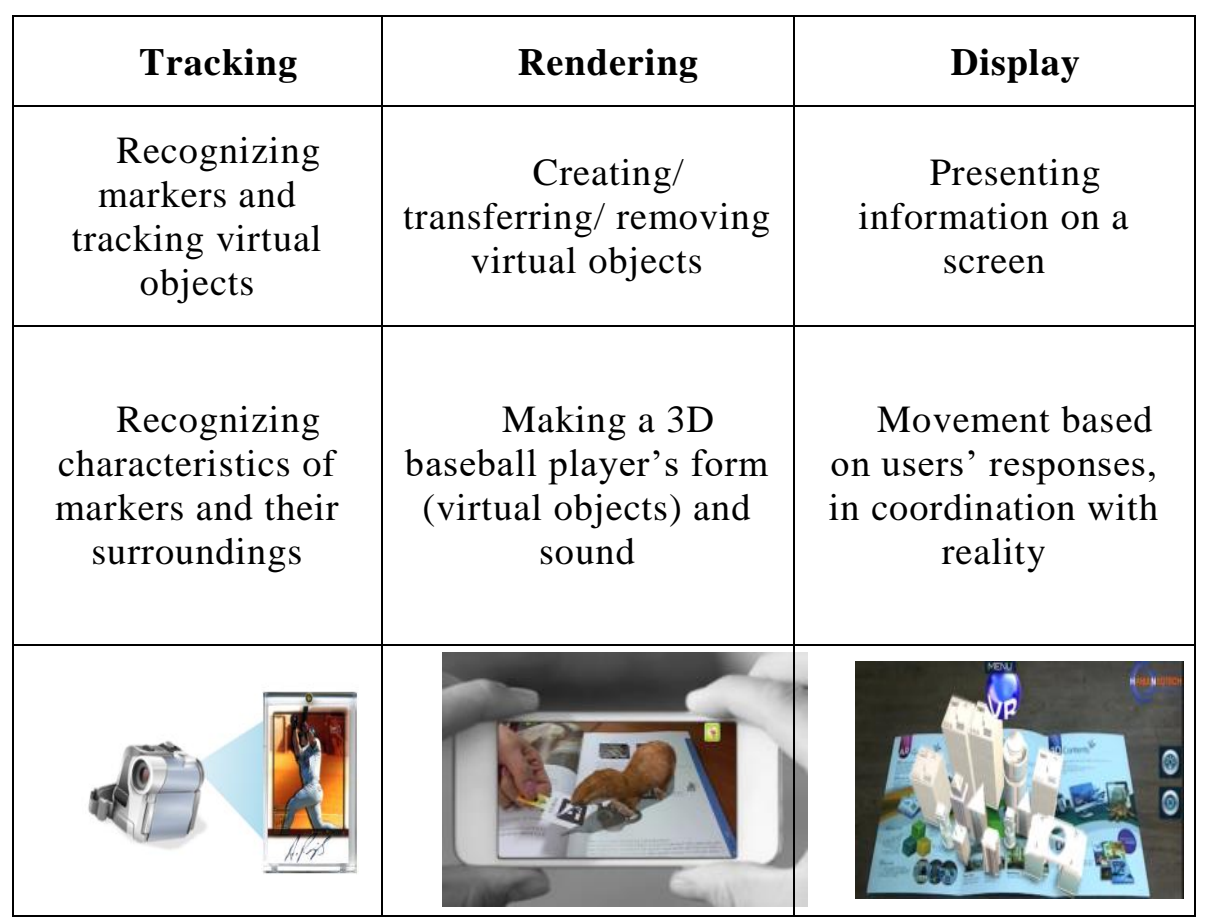

The first research was developing the see-through HMD. Then, Ronald Azuma's design combined images of real-world elements and virtual reality, making it possible for them to interact in real time. Ronald Azuma defines augmented reality as having three features: first, it combines real world elements and virtual reality (the most basic and common characteristics); second, it should be a real-time interaction, and through the users' viewpoints or movements, live information emphasizes an overlap into the real world; and third, it is expressed as 3D images. Considering the previous literature, augmented reality can be defined as the combination of technology together with user interaction [4].

The types of augmented reality are classified into three groups: display, business forms, and representation settings. The first includes handheld displays, see-through HMDs, and projection-based displays, based on the characteristics of the device [6], [9], [10].

Among the different types of display, handheld displays have high mobility and are being actively researched due to increased mobile phone use. See-through HMDs offer the most similar and familiar realization form, namely, the way humans look at objects. These have disadvantages, such as discomfort (e.g., wearing them on your head), and limitations caused by the angles of view. Meanwhile, projection-based displays benefit simultaneous watching, but limit mobility, vision, or distance from placement. The main technologies using augmented reality include tracking, rendering, and display, as seen in Table 2. 
Tracking is a technology of marker recognition for matching virtual objects with the screen, while rendering, on the other hand, is computer-generated imagery adding virtual reality (VR) to the real world. In this process, smooth coordination is quite a significant task, and a virtual object should be focused on the coordinates to lessen visual discordance. Camera adjustment and capturing accurate parameters are needed to synthesize virtual objects in comparable stabilized images in real time. Display technology (presenting information on the screen) is divided into HMD and non-HMD. Augmented reality can be applied to games, advertisements, education, medicine, sightseeing, art, etc. Along with the proliferation of mobile media, interface development is heightened as well.

\section{Current Status of Busan's Tourism Industry and VR}

\subsection{Current Status of Busan's Tourism Industry}

Annual domestic revenue from tourism in 2014 was recorded at approximately US $\$ 18.1$ billion, which increased by $24 \%$ compared to 2013 and was the highest ever. All tourism industries are considered to have a production effect of 33 trillion KRW and to employ about 576,000 people [3,5]. The number of domestic tourists was recorded at about 37 million, which shows an annual average increase rate of $4.9 \%$ over the period from 2009 to 2013. Expenditures by those people was recorded at approximately 23 trillion KRW.

Table 3. Total Number of Foreign Tourists Visiting Busan (In Thousands)

\begin{tabular}{|c|c|c|c|c|}
\hline Year & $\begin{array}{c}\text { Across the } \\
\text { country }\end{array}$ & Busan & Jeju Island & Misc. \\
\hline 2015 & 13,232 & $\begin{array}{c}2,090 \\
(15.8 \%)\end{array}$ & $\begin{array}{c}2,621 \\
(19.8 \%)\end{array}$ & \\
\hline
\end{tabular}

In 2015, the number of cruise ships coming to the port of Busan and the number of people disembarking at the port are expected to total about 131 vessels and 390,000 people (an increase of $11 \%$ compared to 350,000 people the previous year). Since the Pukhang (north port) International Passenger Terminal opened (July 2015) and the Yonghoman Bay Cruise and Nakdong River eco tour cruises were started, Busan marine tourism is expected to strongly advance. Night tours to Busan are also being propelled in several ways: to the Bupyeong night market, the Gwangandaegyo Bridge with lights, and with the top 13 night views of Busan now becoming popular must-visit places for domestic and foreign tourists enjoying night views. Additionally, resting places and observation decks are being built at Hwangnyeongsan Mountain in order to make Hwangnyeongsan Mountain a representative place of Busan because it is good place to enjoy night views of the city properly. Table 3 shows the total number of foreign tourists visiting the city of Busan.

Establishing a basis for smart tourism leads to providing the fundamentals of online marketing, such as developing an intellectual app and websites (which allow tourists to organize tour courses by themselves for individual tours), upgrading a culture tourism home page, and running a Busan advertisement board within the home page of an online tour company headquartered in China. Though the number of Chinese tourists visiting Busan has consistently increased due to an increase in overseas tourists visiting China, with the Korean wave, increased air routes, running chartered planes between Korea and China, and with extended time allowed for transfer tours (from 72 to 100 hours), the increased rate is still lower, compared to nationwide statistics and statistics for Jeju Island. 
To attract more Chinese visitors to Busan, it is necessary to produce dramas or movies in Busan by collaborating with Chinese and Taiwanese movie and drama production companies, and to develop special Busan tour products making use of Korean drama shooting locations. The number of Chinese tourists to Busan, which had shown a tendency to increase thanks to creating new (and improving existing) air routes, had turned to a downward trend because of the weak yen and the Middle East Respiratory Syndrome (MERS) outbreak in 2015. Since September 2015, this downturn has recovered, so the number of overseas visitors to Busan is also increasing.

The Promotion Committee for Developing Tourism was launched on January 1, 2016, to develop potential tourism resources and related products, and an inbound tourism company support plan was undertaken to foster inbound tourism. Since conditions for overseas tourists coming to Busan via cruise ships has improved from opening the Busan International Passenger Terminal (August 2015), and because Chinese tourists prefer tourism via cruise ships, both the number of cruise ships arriving at the port of Busan and the number of people coming through the port are expected to be 226 vessels and 700,000 people in 2016, which will be an increase of three times over the previous year. It is time to develop customized tourism products suitable for tourists on cruise ships and to improve accommodation facilities. It is necessary to attract more Chinese tourists by combining the Korean wave's global killer content with Busan content. Additionally, the Busan One-Asia large-scale festival will be held by linking the Chinese National Day to strengthen Busan's image as a tourism city brand.

Table 4. International Tourists Travelling To Busan Categorized By Nationality (In Thousands)

\begin{tabular}{|c|c|c|c|c|c|c|c|}
\hline Year & Total & China & Japan & US & Russia & UK & Others \\
\hline 2015 & 2,090 & 553 & 368 & 169 & 44 & 36 & 920 \\
\hline 2014 & 3,016 & 922 & 497 & 171 & 69 & 53 & 1,304 \\
\hline 2013 & 2,801 & 781 & 517 & 163 & 66 & 47 & 1,227 \\
\hline
\end{tabular}

In 14 cities and provinces, excluding the metropolitan area centered on Seoul, regulation reforms taking into account unique regional characteristics, fostering a regional strategic industry, and introducing a marine tourism-regulation free zone are urgently necessary. Because of the MERS outbreak in 2015, overseas tourists were recorded at 13.23 million people, a $6.8 \%$ decrease compared to the 14.2 million in 2014. However that tendency is changing. To achieve the objective of a fast-recovering Korean tourism industry and to attract 20 million foreign tourists sooner, the Korean government runs a campaign called $\ulcorner 2016-2018$ Visit Korea Year $\lrcorner$ to make Korea the place where travelers would like to visit again. China is now the biggest outbound market over Japan in the Asia Pacific region. China ranked third in the top long-distance outbound tourism markets and fourth in the top outbound tourism markets in the world. Table 4 shows the current status of foreign tourists visiting Busan by country. 


\section{Tangible Marine Leisure Sports VR System for Busan Region}

\subsection{Tangible Marine Leisure Sports VR System CNDPS}

Figure 2 is the architecture of the tangible marine leisure sports VR system proposed in this study. The system was designed by utilizing Busan's major marine sports tourism items, like the head-mounted display, boat and yacht VR videos, and skin/scuba diving VR videos. The proposed VR system consists of a tangible VR-based smart marine leisure video content platform to maximize Busan's marine leisure-city image specializing in various marine sports, like boats, yachts, windsurfing, skin diving and scuba diving, in addition to offering a tourist place real-image converting platform that allows user intervention and control, a real-time marine leisure interpretation linking platform, an optional marine HMD device, and a mobile VR image content simulation platform at tourist spots.

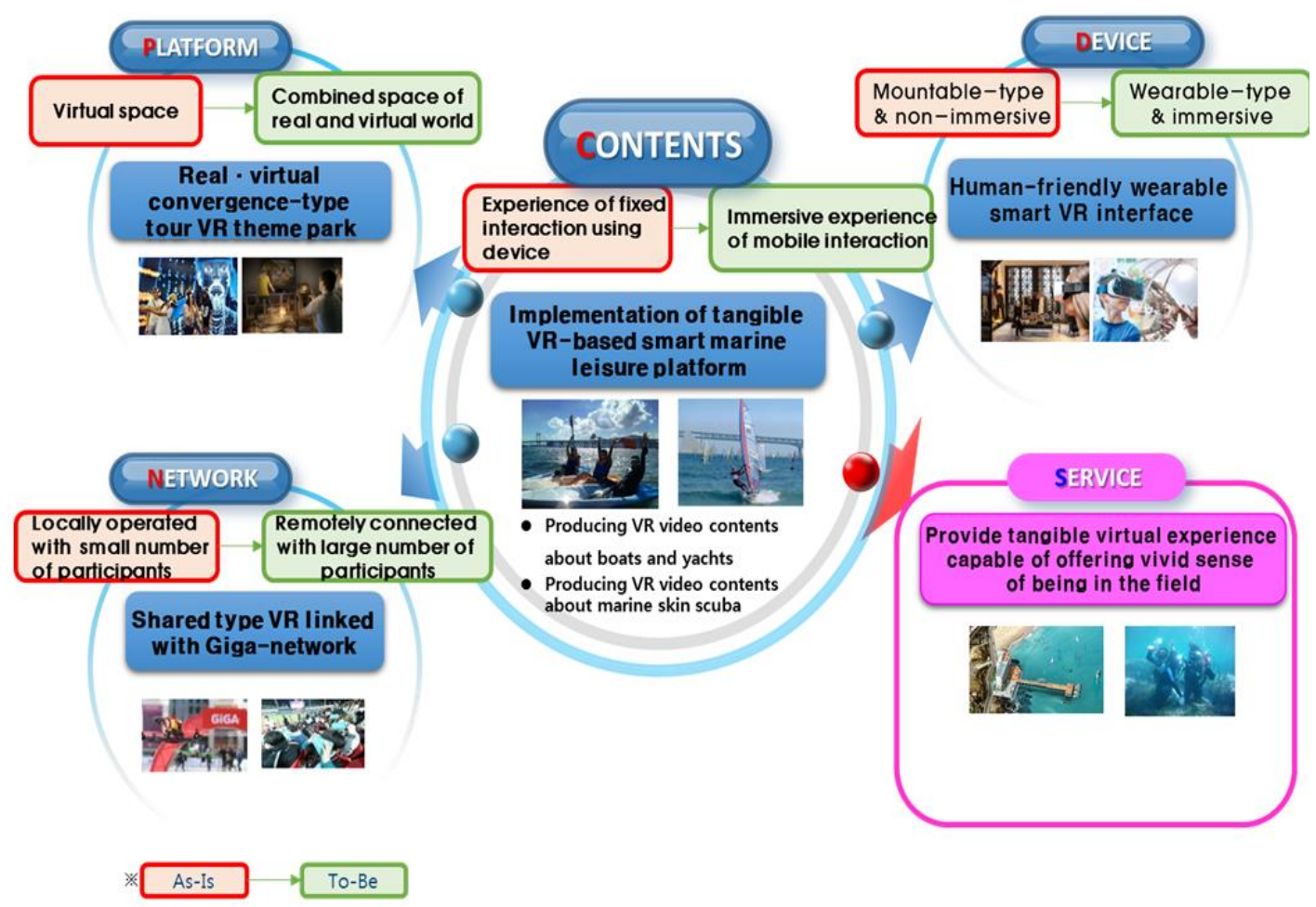

Figure 2. Tangible VR-Based Smart Marine Leisure CNDPS

\subsection{Design and Considerations of Tangible Marine Leisure Sports VR System Content}

Content for the proposed Busan marine leisure sports VR system needs to be designed as tangible VR content in order to make the user feel a sense of immersion and a sense of presence (like standing in real space) by using an HMD and matching the $306^{\circ}$ real scene image taken at the marine tourist place and leisure sports place with user head movement while the user is moving. The contents are designed by taking images of various events and competitions, like the Busan River, the marine sports festival, and the Korea open international dragon boat competitions. Connecting with the Busan Marine Sports Academy and Yacht School, marine leisure sport VR images can be produced. The contents can also be designed as virtual simulation content using the theme of a Busan beach tour for different beaches and yacht competitions. 
There are considerations when tangible marine leisure sports contents are designed. One is transition speed between images, and the other is a residual image problem, both of which are noted as problems in existing VR video content. The frame rate problem and residual images should be fixed. To achieve smoother transition between images, graphics need to be adjusted to maintain 60 to 120 frames per second. To implement a PVR, steps for modeling and mapping video contents through the 3ds Max and Unity 3D engines must be designed. The HMD used for this study is an Oculus Rift device. To ensure a wide-field view, fish-eye rendering with the use of barrel distortion and pincushion distortion effects will be adopted in the HMD for purposes of interpolating distorted images. In particular, when generating video content, it is necessary to pursue a pan-focus in order to make sure the entire screen becomes more clear, while excluding anything out of focus, which makes a specific object be viewed three-dimensionally. In addition, it is necessary to avoid lens dust, motion blur, and run-out effects while taking PVR video for marine leisure sports. Figure 3 shows the screen appearing in the tangible VR-based smart marine leisure video contents system.

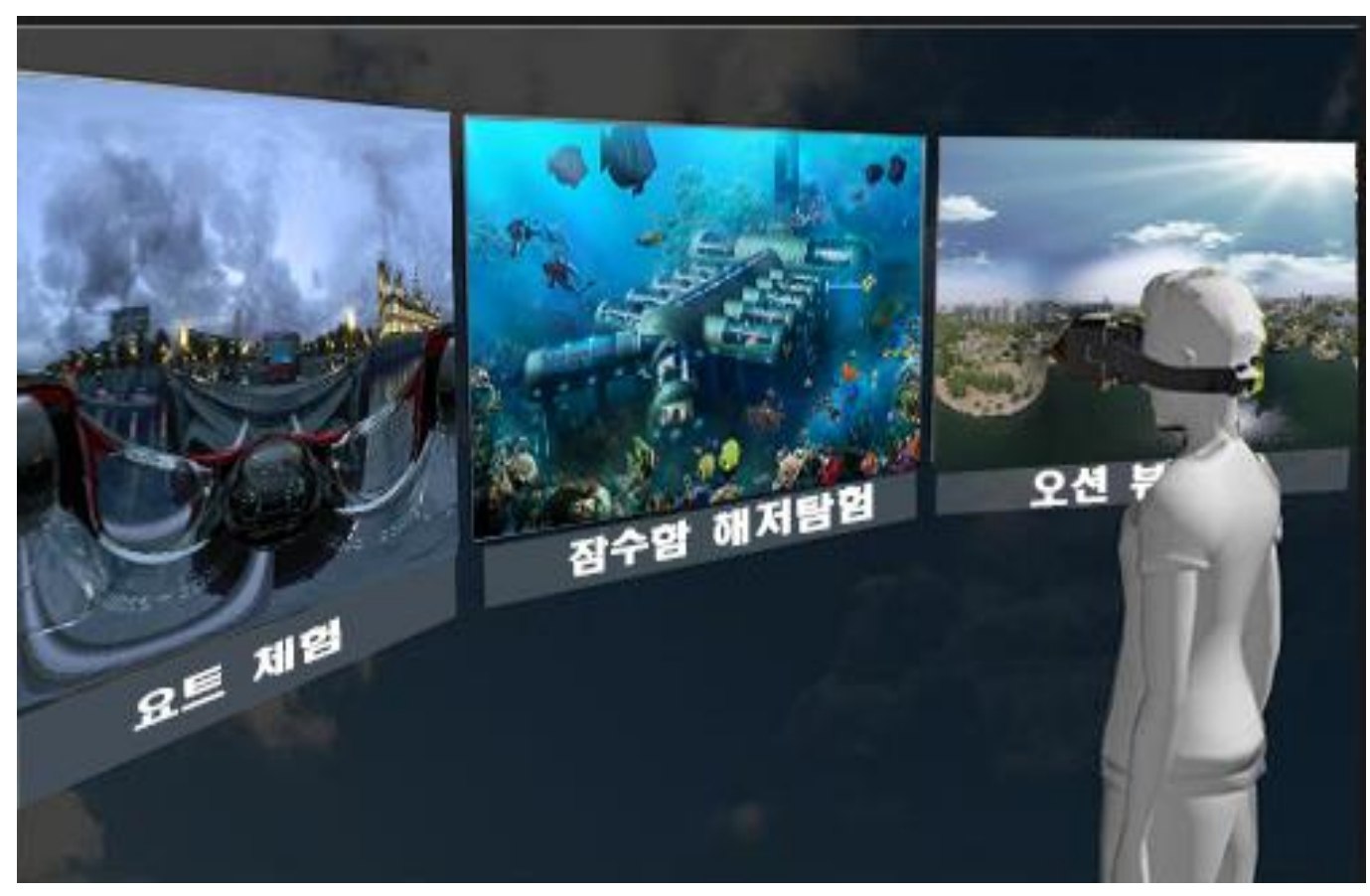

Figure 3. Tangible VR-based Smart Marine Leisure Video Contents System

\section{Conclusions and Future Work}

This paper proposes VR-based tangible content for promoting Busan's tourism industry and marine leisure sports. To this end, the current status of Busan's tourism and tangible culture tourism contents and materials from previous research related to VR were reviewed. Based on the reviews, a tangible VR system that will be applied to Busan's marine leisure sports was suggested. This study is expected to contribute to increasing commercialization of VR technology-based tourism content by means of convergence technology that enables users to perceive active and autonomous marine sports experiences for a flourishing tourism industry in Busan.

Future work will be making practical use of the VR system in playing disaster safety videos and cinema videos considering Busan's regional uniqueness. In terms of the range of contents, more efforts need to be put into making storyboards of videos for enhancing a sense of immersion and making a disaster safety storyboard. 


\section{References}

[1] J.S. Han and G. H. Lee, "VR Tourism Content Using the HMD Device", Journal of the Korea Contents Association, vol. 15, no. 3, (2015), pp. 40-47, 2015.

[2] http://www.gartner.com, "Hype Cycle for Emerging Technologies".

[3] H.G. Lee and J.W. Han, "A Advanced Research of the Range of Marine Leisure Sports and Improvement Design of Relevant Facility: Mainly with Puasn Suyoung bay”, Journal of Digital Design, vol.13, no. 2, (2013), pp. 285-294.

[4] J.C. Kim, E. K. Kim, S. H. Kim, J. W. Park, D. K. Kim and H. S. Lee, "Selection Module Q-sorting System of Augmented Reality-based Physical Activity for Developmental Disability Children", International Journal of u- and e- Service, Science and Technology, vol.10, no. 6, (2017), pp.137-148.

[5] H.G. Lee, "A Study on User Access Convenience of Marine Leisure Sports on Design", Journal of Korea Design Knowledge, vol.29, (2014), pp. 365-375.

[6] V. Teichrieb, "A Survey of Online Monocular Markerless Augmented Reality", International Journal of Modeling and Simulation for The Petroleum Industry, vol. 1, no. 1, (2007), pp. 1-7.

[7] J. I. Lee and S. H. Yoo, "Implementation and Application of Fairy-Tale Book of an Augmented Reality Based on Smartphone and Web Browser", Journal of Korea Association of Information Education, vol. 16, no. 2, (2011), pp. 201-209.

\section{Authors}

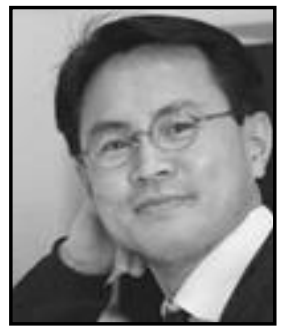

Kim Cheeyong, he received a $\mathrm{BSc}$, an $\mathrm{MSc}$ and a $\mathrm{PhD}$ from Inje University, Republic of Korea, in 1991, 1994, and 2000, respectively. He was a visiting professor at Oxford University in 2007, and a visiting professor in the Digital Clothing Center at Seoul National University in 2012. Currently, he is a professor at the College of ICT, Major of Visual Game Engineering, at DongEui University. Prof. Kim held private exhibitions four times, in both Seoul and China, and has participated in more than 230 international group exhibitions $\mathrm{He}$ has keen interests in 3D animation, fractal \& chaos design, computational simulations, game character design, and 3D virtual fashion fitting systems.

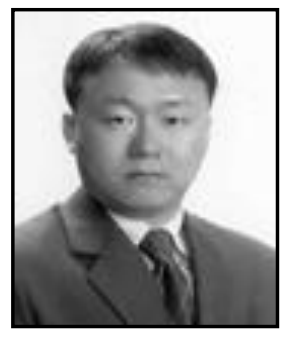

Jong-Chan Kim, he received his BSc from Sunchon National University in 2000, his MSc from the Department of Computer Science, Sunchon National University, in 2002, and his PhD from the Department of Computer Science, Sunchon National University, in 2007. He has been a senior research professor in the Automation and System Research Institute at Seoul National University since 2013. His current research interests are image processing, computer graphics, digital clothing, AR, and games.

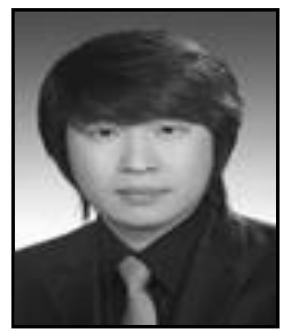

Se-Hoon Jung, he received his BSc and MSc in Multimedia Engineering from Sunchon National University in 2010 and 2012, respectively. Currently, he is a team manager with the research \& development team, Gwangyang Bay SW Convergence Institute, South Korea. His research interests include data analysis and data prediction. 


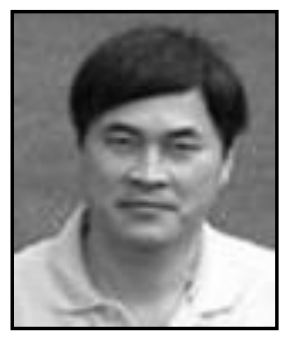

Eung-Kon Kim, he received a BSc from Chosun University, Gwangju, Korea, in 1980. His MSc was from the Department of Electronics at Hanyang University in Seoul, Korea, in 1987. His PhD was from Chosun University in Gwangju, Korea, in 1992. His current research interests are computer vision, virtual/augmented reality, image processing, and computer graphics. Currently, he is a professor in the Department of Computer Engineering, Sunchon National University, Korea. 